\title{
Ulrike Haß
}

\section{Semantische Umgebung und Mitspieler}

1. Eine ungewohnte Angabeart 227

2. Argumentstruktur und Frame-Semantik 228

3. Methodisches 230

4. Literaturverzeichnis 233

\section{Eine ungewohnte Angabeart}

„Semantische Umgebung und lexikalische Mitspieler" heißt in elexiko eine Angabeart, die es erst in wenigen Wörterbüchern überhaupt gibt und deren Funktion für Nutzer und Nutzungssituationen im Grunde noch unbekannt ist. Auf jeden Fall können sie daraus etwas Interessantes über Wörter und Wortschatz lernen. In elexiko findet man über den Klick auf den entsprechenden Reiter beim Stichwort mobilisieren (das nur eine Lesart hat) etwa Folgendes:

Wer mobilisiert?

Betriebsrat

Gewerkschaft

Körper

Mannschaft

Medien

Opposition

Partei

Regierung
Wer oder was wird mobilisiert?

Anhänger

Basis

Energien

Gelder

Kräfte

Leute

Massen

Sponsoren

Unterstützung

Volk

Wähler

Widerstand 


\section{Argumentstruktur und Frame-Semantik}

Diese Fragen vertreten jeweils eine der semantischen Rollen des Stichworts; die Antworten zeigen die Realisierung der Rollen im elexiko-Korpus (s. P. Storjohann, Das elexiko-Korpus, in diesem Band). Man könnte sagen, dass hinter dieser Angabeart die Argumentstruktur mit den semantischen Rollen steht. In elexiko werden die Argumente lexikalischer Ausdrücke in dieser Angabe aber semantisch, nicht syntaktisch aufgefasst - Satzbaupläne und Valenzangaben werden ja bereits bei der Grammatik präsentiert, wohingegen die Angaben zu semantischer Umgebung und lexikalischen Mitspielern in unmittelbarem Zusammenhang mit der Bedeutungserläuterung angeboten werden. Statt als Argumentstruktur und semantische Rollen kann man diese Angabeart linguistisch also auch oder eher unter dem Aspekt von Frames sehen (vgl. Wegener 1989). Die Fragen repräsentieren dann die Slots und die aus dem Korpus gewonnenen Antworten stehen für die Filler oder Partizipanten. Die syntaktische Relation zwischen Stichwort und Fillern spielt hier keine Rolle; deshalb sind die Realisierungen der Filler in elexiko auch unflektiert in ihrer Normal- oder Nennform angegeben. Pluralformen jedoch können vorkommen, da viele Wörter der öffentlichen Diskussion nicht nur öfter pluralisch als singularisch gebraucht werden, sondern auch, weil der Plural semantisch nicht selten vom Singular abweicht.

Die Beiträge in Hummel/Kailuweit (2004) zeigen ein breites Spektrum semantiktheoretischer Positionen in der neueren Diskussion um semantische Rollen (vgl. bes. Jacob 2004, Kailuweit 2004, Primus 2004, Schneider 2004, Wotjak 2004). Vor diesem Hintergrund gesehen liegt der den Mitspieler-Angaben in elexiko zugrundeliegende Ansatz - wegen der Komplementarität zu den elexiko-Valenzangaben - weitestgehend entfernt von einer syntaktischen Interpretation der Argumentstruktur und näher an einer wort- und letztlich diskurssemantischen Auffassung. Die Angaben zu „Semantischer Umgebung und Mitspielern" (so die für elexiko gewählte, dem Laienverständnis zugängliche Bezeichnung in der Online-Ansicht) sollen einen bestimmten Typ kognitiver Assoziationen zwischen dem Stichwort in einer Lesart und Wörtern in dessen Textumgebung darstellen. Dabei ist der empirische Zugang zur Textumgebung qua Korpus entscheidend. Es hat sich gezeigt, dass die Listen der signifikanten Kookkurrenzpartner (s. U. Haß, elexiko - Das Projekt, in diesem Band) Lexeme enthalten, die sich als Realisierung semantischer Rollen auffassen lassen, und zwar unabhängig von der Wortart.

Die Angabe der semantischen Umgebung und ihrer lexikalischen Mitspieler in elexiko ist daher keineswegs auf Verben oder nominale Handlungsprädikatoren beschränkt. Z. B. findet man unter Mobilität in der Lesart ,Erreichbarkeit' folgendes: 


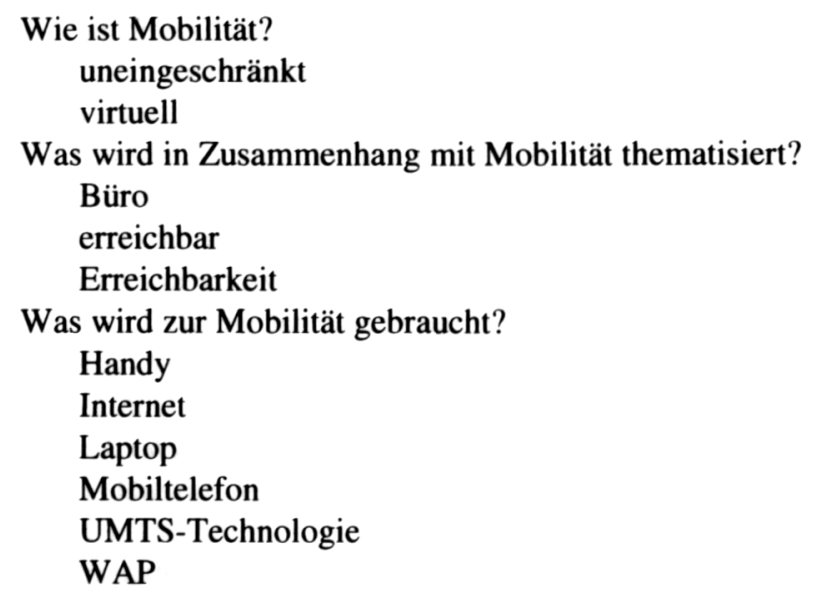

Welche Slots, d. h. welche Art von Fragen bei einer Lesart angesetzt werden, hängt von der semantischen Klasse des Stichworts ab (vgl. U. Haß, Das Bedeutungsspektrum, in diesem Band). Wie erwähnt, sind es nicht nur Verben, bei denen sich nach den Partizipanten fragen lässt, sondern alle anderen Autosemantika und teilweise sogar Synsemantika; bei Letzteren müssen die Fragen allerdings entsprechend umformuliert werden. Bei abzüglich ${ }^{l}$ z. B. zeigt das Korpus eine signifikante Reihe von Mitspielern wie Aufwendungen, Ausgaben, Inflationsrate, Rabatt, Schulden, Tilgung zu denen sich die Frage „Was wird abgezogen?" stellen lässt. Weitere Slots von abzüglich repräsentieren die Fragen „Wovon wird abgezogen?“ (Filler: Ertrag, Börsenkurs, Gewinn, Verkaufspreis u. a.) und „Was ist das Ergebnis des Abziehens?“ (Filler: Bruttospielertrag, Nettoschuld $\mathrm{u}$. a.).

Die elexiko-Redaktionsrichtlinien enthalten zu jeder semantischen Klasse ein Standardrepertoire von Fragen. Die Fragen vertreten die Namen linguistischer Kategorien wie Agens, Instrument, Patiens usw. Unsere strikt semantische, von syntaktischer Realisierung unabhängige Auffassung der Rollen erlaubt die Einbeziehung von so etwas wie diskursiven Rollen, typischerweise bei der Frage „Was wird in Zusammenhang mit X thematisiert?" Die LexikografInnen finden und formulieren die Fragen im Zuge einer Klassifikation der im Korpus ermittelten Kookkurrenzpartner. Dabei ergibt sich induktiv erwartungsgemäß ein Standardrepertoire an Fragen, das dem bekannten, lateinisch oder englisch benannten Kategorieninventar entspricht, das aber zugleich offen und flexibel gehalten werden kann und muss, um dem usuellen Gebrauch der Wörter im Korpus so nah wie möglich zu kommen.

Dieser Wortartikel ist derzeit noch nicht online veröffentlicht. 
Der Vorteil dieses Verfahrens, das ähnlich auch von Konerding (1993) entwickelt wurde, ist, dass natürlichsprachliche Fragen nutzerfreundlicher sind als lateinische Ausdrücke wie „Agens“ oder englische wie „Experiencer“. Aber auch das bekannte und in den Beiträgen von Hummel/Kailuweit (2004) mehrfach reflektierte Problem eines geschlossenen Kategorieninventars wird damit umgangen. Einige wenige, sehr allgemeine Protorollen anzusetzen (vgl. Primus 2004), führt in der lexikografischen Praxis leicht zu trivialen Aussagen. Ein größeres, aber geschlossenes Kategorieninventar hat gerade Lexikografen vor unüberwindliche Schwierigkeiten gestellt, wie Greule (1997) anhand des Versuchs zeigt, ahd. Verben mit Tiefenkasus zu beschreiben. Ein korpusbasierter, empirischer Zugang von den Texten her sollte - so die elexiko-Überlegung - einen anderen, d. h. nutzerfreundlicheren und empirienäheren Weg wählen.

Die natürlichsprachlichen Fragen bilden in elexiko eine unabgeschlossene Menge mit standardisiertem Kernbestand. Von dem Standardrepertoire dürfen die Lexikografen immer dann abweichen, wenn die Korpusbefunde Filler für eine „neue“ Art von Slot offenbaren. Die Fragen zeigen also die Struktur der im Korpus typischen und durch Frequenz ausgewiesenen semantischen Umgebung, oder, wie wir es in der Projekt-Homepage (www.elexiko.de) erklären: Die Fragen zeigen das semantische Netz, in dessen Mitte das Stichwort in seiner jeweiligen Lesart sitzt. Dieses semantische Netz liegt auf der syntagmatischen Ebene und damit quer zum Netz der paradigmatischen Relationen; beide ergänzen einander. Die Empirie hat auch hier Vorrang, und so stellt sich schon einmal heraus, dass bei einem fast ausschließlich passivisch gebrauchten Handlungsprädikator die Agensrolle auch semantisch peripher wird. D. h. dass die empirische Beschreibung der Frames in Fragen und Antworten auch theoretisch relevant werden kann, insofern sie es erlauben, die Distribution bestimmter Rollen auf die semantischen Klassen zu überprüfen, vorausgesetzt, in elexiko sind nicht nur wenige hundert, sondern ein paar tausend Wörter beschrieben. Dies wird in einigen Jahren der Fall sein.

\section{Methodisches}

Oben wurde erwähnt, dass Menge und Art der in elexiko verzeichneten lexikalischen Mitspieler, der Filler-Realisierungen, mithilfe der Cosmas-Kookkurrenzanalyse zustande kommen. Nachfolgend soll erläutert werden, wie und worin sich die Mitspielerangaben von der Angabeart der ,typischen Verwendungsmuster" unterscheiden (vgl. dazu, P. Storjohann, Typische Verwendungen, in diesem Band). 
Der elexiko-Wortartikel Zukunft in der Lesart ,kommende Zeit ' enthält unter der Überschrift „Semantische Umgebung und lexikalische Mitspieler“ folgende Angaben:

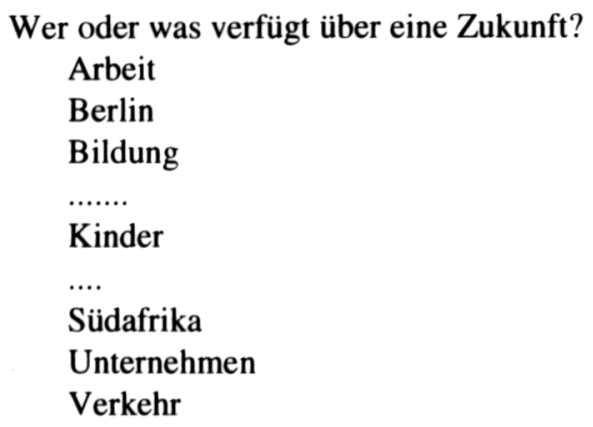

Eine der die Slots kennzeichnenden Fragen lautet „Wer oder was verfügt über eine Zukunft?“. In der Liste der Filler-Realisierungen findet man Kinder - der Plural ist hier semantisch wichtig. Dieser Eintrag wird methodisch aus der Liste der signifikant häufigen Kookkurrenzpartner zu Zukunft abgeleitet, die mit der Cosmas-Kookkurrenzanalyse ermittelt wird. Von der Liste der Kookkurrenzpartnerlexeme aus lassen die Lexikografen sich die jeweiligen Kotexte anzeigen, die Stichwort und Partnerwort umspannen; dies sind z. B.:

\section{Sicherung der Zukunft unserer Kinder eine Zukunft für die Kinder}

Kinder, die die Zukunft der Gesellschaft sind. die Zukunft meiner drei Kinder

\section{Kinder ohne Zukunft?}

Die kleinen Kinder brauchen eine abgesicherte Zukunft

Zukunft für alle Kinder

Die Zukunft dieser „wehrlosen“ Kinder

um unserer Kinder willen, deren Zukunft

des Fördervereins ,Zukunft für Kinder“

Das Beispiel soll zeigen, dass zwischen zwei Lexemen eine lexikalisch-semantische Relation auf der syntagmatischen Ebene existiert, die unabhängig von bestimmten syntaktischen Relationen ist. Die o. g. Kotextausschnitte sind heuristisch zu nutzendes Material, anhand dessen Lexikografen die typischen Realisierungen einer semantischen Rolle überprüfen. Die Nutzer sehen lediglich die mit Ausnahme des Plurals unflektierten Lexeme, zwischen denen eine entsprechende semantische Verknüpfung besteht. 
Im Unterschied hierzu zeigen die Angaben unter der Überschrift „Typische Verwendungen" die im Korpus nachweisbaren typischen syntagmatischen Muster zum Stichwort in einer Lesart. D. h., hierbei handelt es sich um Kollokationsbeziehungen im eigentlichen Sinne, bei denen die syntaktischen Beziehungen nicht ausgeblendet werden dürfen und die darüber hinaus mehr als Stichwort und Partnerwort umfassen können. Zu Zukunft in der Lesart ,kommende Zeit' finden die Nutzer z. B. folgende typische Verwendungen:

\author{
in nächster Zukunft \\ in naher Zukunft \\ Visionen der Zukunft \\ an die Zukunft denken \\ Diskussion über die Zukunft \\ die berufliche Zukunft sichern \\ Investition in die Zukunft \\ in Zukunft enger zusammenarbeiten \\ langfristig seine Zukunft planen \\ Lösungen für die Zukunft \\ Sorge um die Zukunft \\ zuversichtlich in die Zukunft schauen \\ sich Gedanken über die Zukunft machen \\ sich etwas für seine Zukunft wünschen \\ sich über seine Zukunft Gedanken machen \\ [...] soll in Zukunft verstärkt werden \\ auf dem Weg in eine bessere Zukunft
}

Bei der Angabeart „Semantische Umgebung und lexikalische Mitspieler" besteht die lexikografische Arbeit an und mit den Kookkurrenzlisten nun darin, die für jede Lesart typischen Filler-Lexeme nach ihren Rollen oder Slots zu klassifizieren und die Frage zu formulieren, auf die jede gefundene Klasse die Antwort liefert. Oft sind die Listen der Filler-Lexeme zu umfangreich; wenn eine Auswahl nötig wird, wird diese in einem Kommentar begründet. Wenn z. B. ein Agens durchgehend durch Personennamen realisiert wird, wäre es wenig sinnvoll, eine Liste von Personennamen aufzuführen. Aber in einem Kommentar wird auf eine solche typische Realisierung hingewiesen. Wenn die Liste lauter Determinativkomposita und auch deren Grundwort enthält, wird nur das Grundwortlexem ausgewählt. Auch dazu erfolgt ein Kommentar. Kommentare werden auch dann gegeben, wenn die Kookkurrenzanalyse keine Mitspieler erkennen lässt, was vor allem bei Stichwörtern unter 100 Treffern 
der Fall ist. Hier werden exemplarische Mitspieler ausgewählt, im Kommentar wird aber festgehalten, dass diese ohne statistische Signifikanz sind.

Aus dem korpusstatistischen Verfahren resultiert auch, dass die Angabe der semantischen Umgebung bei niedrigfrequenten Lexemen in aller Regel fehlt. Die automatische Kookkurrenzanalyse macht hier keinen Sinn. Bei niedrigfrequenten Lexemen werden die Belege und ihre Veranschaulichungspotenz wichtiger.

\section{Literaturverzeichnis}

Greule, Albrecht (1997): Probleme der Beschreibung des Althochdeutschen mit Tiefenkasus. Ein Erfahrungsbericht. In: Semantik der syntaktischen Beziehungen. Akten des Pariser Kolloquiums zur Erforschung des Althochdeutschen 1994, hg. von Yvon Desportes. Heidelberg. S. 107-122.

Hummel, Martin/Kailuweit, Rolf (2004): Einführung: Semantische Rollen in der Diskussion. In: Kailuweit, Rolf/Hummel, Martin (Hg.): Semantische Rollen. Tübingen. S. IX-XXIX. (Tübinger Beiträge zur Linguistik; 472).

Jacob, Daniel (2004): Generalisierte Semantische Rollen und freie Linkingprinzipien. In: Kailuweit, Rolf/Hummel, Martin (Hg.): Semantische Rollen. Tübingen. S. 104-117. (Tübinger Beiträge zur Linguistik; 472).

Kailuweit, Rolf (2004): Protorollen und Makrorollen. In: Kailuweit, Rolf/Hummel, Martin (Hg.): Semantische Rollen. Tübingen. S. 83-103. (Tübinger Beiträge zur Linguistik; 472).

Konerding, Klaus-Peter (1993): Frames und lexikalisches Bedeutungswissen. Tübingen. (Reihe Germanistische Linguistik; 142).

Primus, Beatrice (2004): Protorollen und Verbtyp: Kasusvariation bei psychischen Verben. In: Kailuweit, Rolf/Hummel, Martin (Hg.): Semantische Rollen. Tübingen. S. 377-401. (Tübinger Beiträge zur Linguistik; 472).

Schneider, Franz (2004): Lexikologische Studie auf der Grundlage des „Ereignisbegriffs“ - aufgezeigt am Beispiel ,fusion d'entreprises'. In: Kailuweit, Rolf/Hummel, Martin (Hg.): Semantische Rollen. Tübingen. S. 447463. (Tübinger Beiträge zur Linguistik; 472).

Wegener, Immo (1989): Lexikographische Definition und Frame-Theorie im allgemeinen einsprachigen Wörterbuch. In: Wörterbücher. Ein internationales Handbuch zur Lexikographie. 3 Teilbde. Hg. v. Franz Josef Hausmann, Oskar Reichmann, Herbert Ernst Wiegand, Ladislav Zgusta, Berlin/New York 1989, 1990, 1991, 1. Teilbd., S. 893-899.

Wotjak, Gerd (2004): Partizipantenrollen in Sachverhaltswissensrepräsentationen und als semantisch-funktionale Argumentbestimmungen in Verbbe- 
deutungen. In: Kailuweit, Rolf/Hummel, Martin (Hg.): Semantische Rollen. Tübingen. S. 3-36. (Tübinger Beiträge zur Linguistik; 472). 